\title{
Diet-Microbiota-Brain Axis in Alzheimer's Disease
}

\author{
Halle J. Kincaid ${ }^{a}$ Ravinder Nagpal ${ }^{b}$ Hariom Yadava \\ aDepartment of Internal Medicine-Molecular Medicine, Wake Forest School of Medicine, Winston-Salem, NC, USA; \\ ${ }^{b}$ Department of Nutrition, Food \& Exercise Sciences, Florida State University, Tallahassee, FL, USA
}

\section{Keywords}

Amyloid · Cognition · Dementia · Microbiota .

Neurodegenerative disease $\cdot$ Tau

\begin{abstract}
Background: Alzheimer's disease (AD) is the most common form of dementia, particularly in older adults, with clinical manifestations of progressive cognitive decline and functional impairment. The prevalence of AD and related dementia is mounting worldwide, but its etiology remains unresolved, with no available preventative or ameliorative therapy. Emerging evidence suggests that the gut microbiota of patients with AD is different from cognitively normal counterparts. Summary: Communication between gut and brain (gut-brain axis) plays a crucial role in AD pathology. Bacteria inhabiting the gut strongly influence this gut-brain axis and thus may participate in AD pathology. Diet, one of the strongest modulators of gut microbiota, also strongly influences brain health and AD pathology. Gut microbiota metabolites including short-chain fatty acids, pro-inflammatory factors, and neurotransmitters may also affect AD pathogenesis and associated cognitive decline. Therefore, investigation of diet-microbiota-brain axis is important to better understand its contribution in AD pathology and its potential use as a
\end{abstract}

target to prevent and treat AD. Herein, we discuss the link between $A D$ and gut microbiota and ponder how microbiota modulation through nutritional approaches may offer avenues for discovering novel preventive and therapeutic strategies against AD. Key Message: A strong association exists between lifestyle factors and AD prevalence wherein unhealthy dietary factors have been linked to neurodegeneration. Specific prudent dietary patterns might help in preventing or delaying $A D$ progression by affecting $\beta$-amyloid production and tau processing and regulating AD-associated inflammation, metabolism and oxidative stress, plausibly via modulating gut microbiota.

(c) 2021 The Author(s).

Published by S. Karger AG, Basel

\section{Introduction}

Alzheimer's disease ( $\mathrm{AD})$ is a neurodegenerative disorder affecting over 50 million people worldwide and is the leading cause of dementia and cognitive decline, especially in older adults [1]. Antecedently, AD prevalence was highest in high-income countries (West Europe and

Halle J. Kincaid and Ravinder Nagpal contributed equally to the manuscript.

karger@karger.com www.karger.com/anm

Karger $\stackrel{\text { ' }}{=}$

BOPEN ACCESS
(C) 2021 The Author(s)

Published by S. Karger AG, Basel

This is an Open Access article licensed under the Creative Commons Attribution-NonCommercial-4.0 International License (CC BY-NC) (http://www.karger.com/Services/OpenAccessLicense), applicable to the online version of the article only. Usage and distribution for commercial purposes requires written permission.
Correspondence to:

Ravinder Nagpal, rnagpal@fsu.edu

Hariom Yadav, hyadav@wakehealth.edu 
North America); however, dementia incidences have started to increase in middle- to low-income countries, with a projected $63 \%$ of all cases being in middle- to lowincome countries by 2030 and $71 \%$ of all cases by 2050 [1]. AD majorly affects the elderly ( $>65$ years), indicating aging as one of the major risk factors, where aging-associated $\mathrm{AD}$ progression is called sporadic $\mathrm{AD}$ [2]. While the reasons for increasing $\mathrm{AD}$ prevalence remain unclear, emerging evidence has linked dietary habits to the $\mathrm{AD}$ pathology [3]. For instance, obesity and type- 2 diabetes are potential risk factors for $\mathrm{AD}$, and studies have linked high-fat diet and high-carb diet with the risk of AD development. Conversely, the Mediterranean (MD) and ketogenic diets have been linked with healthier brain aging and lower AD risk [4-7]. Further corroborating this evidence, there is an increase in $\mathrm{AD}$ prevalence following the epidemiological shift seen in developing countries where there are increasing proportions of obese and diabetic people [1]. In addition to these macroscopic issues, bacteria living in our gut (gut microbiota) may play an important role in $\mathrm{AD}$ pathology $[2,8-10]$. While mechanisms are still not fully understood, studies suggest that the gut microbiota may influence the brain via inflammation, neurotransmitters, and hormones. Thus, reversing gut microbiota abnormalities through healthy diets could benefit the brain and reduce $\mathrm{AD}$ risk. Herein, we discuss current research linking diet and gut microbiota with $\mathrm{AD}$ pathology based on the gut-brain axis.

\section{The Pathophysiology of AD}

Although the pathology and contributing factors for $\mathrm{AD}$ remain unclear, there are genetic mutations such as the extra copy of the amyloid precursor protein (APP) gene and trisomy of chromosome 21 , which enhance the neurodegenerative amyloid-beta $(\mathrm{A} \beta)$-protein deposition associated with familial AD [11]. Pathologically, extracellular neurofibrillary plaques and intracellular hyperphosphorylated tau (pTau) tangles are found throughout the cortical parenchyma of the $\mathrm{AD}$ brain, especially in the temporal lobes [12]. Profound cerebral atrophy may also support the diagnosis of $\mathrm{AD}$. These visible characteristics have been viewed as main factors of $\mathrm{AD}$ pathology for a long time. The buildup of $A \beta$ plaques and neurofibrillary tangles of pTau in the neocortex causes inflammation, oxidative stress, and ultimate neurodegeneration. These symptoms are also mediated by reactive oxygen species, which are normally kept in check via enzymatic antioxidants. However, when antioxidant levels decrease, reac- tive oxygen species can cause oxidative stress and lead to neurodegeneration [13]. The inflammation in the brain is also linked with gut leakiness that is common in $\mathrm{AD}$ patients, where immune-system agents such as polymorphonuclear neutrophils are able to leak out of the intestinal walls. Continual inflammation from the gut can start to degrade the blood-brain barrier, allowing these inflammatory agents to enter the brain and cause further inflammation. Biomarkers used in $\mathrm{AD}$ diagnosis include low $\mathrm{A} \beta$ 42 and high tau levels in the brain, decreased fluorodeoxyglucose uptake on PET, and structural cerebral atrophy on MRI. The clinical diagnosis of $\mathrm{AD}$ varies from patient to patient and is difficult to characterize with a prescribed set of symptoms. However, symptoms that are more severe than those typical of old age in the ten major diagnostic categories can be a red flag for diagnosticians. These 10 areas of concern in the $\mathrm{AD}$ patient are: degradation/difficulty with memory, problem-solving, familiar task-completion, time/place recognition, comprehension of images or spatial relationships, communication, retracing steps, judgment/interpersonal interactions, work/social activities, and mood/personality [14].

Because of incomprehensibility of amyloid cascade hypothesis, studies are investigating $\mathrm{A} \beta$ plaques and $\mathrm{pTau}$ as markers of a system that has been disrupted from many ends. Studies are hypothesizing that $\beta$-amyloids are antimicrobial peptides which may accumulate in the $\mathrm{AD}$ brain due to higher carriage of nonbeneficial microbes possibly coming from the degraded blood-brain barrier resulting from inflammation [2]. These amyloids are formed by the cleavage of APP and the activation of myeloid differentiation primary response 88 pathway by tolllike receptor 2 [2]. In addition to brain amyloidosis, there are bacterial amyloids located in the gut, which resemble CNS amyloids in tertiary structure and might play a role in stimulating the immune system in the brain as the immune system learns to recognize the amyloids in the gut and then has an enhanced attack of the amyloids in the brain, leading to inflammation. The leaky gut syndrome that allows for inflammation and neurodegeneration in $\mathrm{AD}$ is further mediated by diet, for example, high-fat diet. Thus, the measurable symptoms of $\mathrm{AD}$ have complex causes that correlate with gut microbiota.

\section{Gut-Brain Axis in AD}

The $A \beta$ plaque and tau tangles hypotheses were the ideal model for AD pathophysiology. However, emerging research has established $\mathrm{AD}$ as a component of systemic
Kincaid/Nagpal/Yadav 


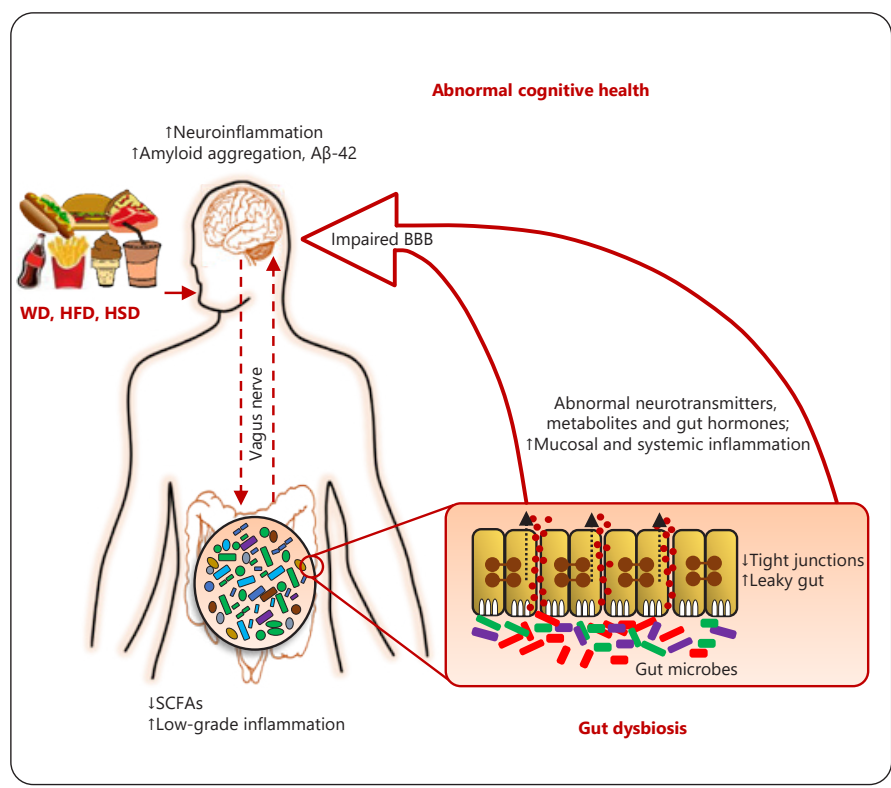

Fig. 1. Schematic representation of the putative links connecting diet-microbiome interaction with brain and cognitive health in subjects with $\mathrm{MCI}$ and $\mathrm{AD}$. $\mathrm{A} \beta$, amyloid-beta; $\mathrm{BBB}$, blood-brain barrier; BDNF, brain-derived neurotrophic factor; DASH, dietary approaches to stop hypertension diet; HFD, high-fat diet; HSD,

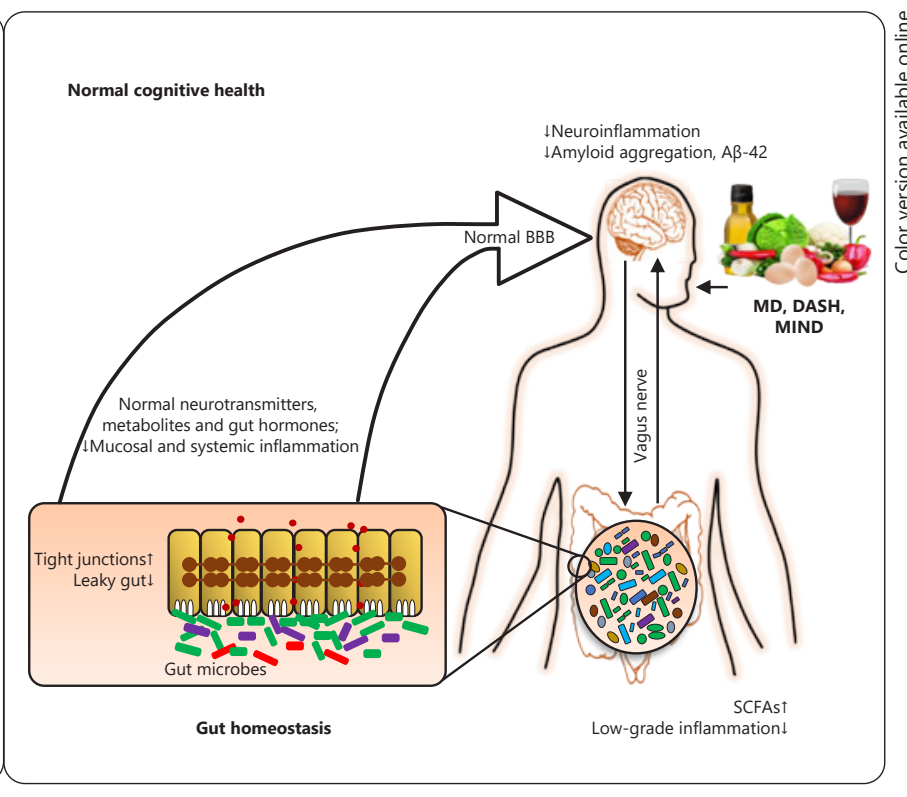

high-sugar diet; MD, Mediterranean diet; MIND, MediterraneanDASH Intervention for Neurodegenerative Delay diet; NMDAR, N-methyl-D-aspartate receptor; SCFA, short-chain fatty acids; WD, Western-style diet; AD, Alzheimer's disease; MCI, mild cognitive impairment. dysfunction at least in-part mediated by chronic, systemic, and neuronal inflammation, along with gut microbiota. Within this, neuroinflammation hypothesis lies the gut-brain axis, which links the gut microbiota activities to neuronal health and dysfunction (Fig. 1). The effects of gut microbiota in $\mathrm{AD}$ are mediated by microbial metabolites that either act on local neurons in the gut and surrounding tissues and send signals to the brain, and/or get absorbed from the gut and reach to brain through circulation. Such examples are monoamines, short-chain fatty acids (SCFAs), gamma-aminobutyric acid (GABA), betamethylamino-L-alanine, brain-derived neurotrophic factor, serotonin, and dopamine $[8,10]$.

Emerging human studies including ours have revealed distinct microbiota patterns in $\mathrm{AD}$ patients and/or subjects with mild cognitive impairment (MCI) $[7,9]$. The already-present microbiota dysbiosis further impacts the aging brain as the natural aging-related gut dysbiosis begins to develop and weakens the intestinal epithelia, causing gut leakiness and inflammation [15]. Increased systemic inflammation instigates immune impairments, allowing $\beta$-amyloid buildup in the brain [15]. This indicates that patients typically develop $\mathrm{AD}$ during old age even when risk factors were present from early life, for example, genetic mutation. Patients with $\mathrm{MCI}$ and $\mathrm{AD}$ also show higher levels of gut microbiota-derived trimethylamine N-oxide (TMAO) in the cerebrospinal fluid [16], which correlate with AD biomarkers including pTau, total Tau, and A $\beta 42$. TMAO treatment reduces cognitive function and aging signs in mice, by ameliorating neuronal senescence and mitochondrial dysfunction [17]. TMAO and its precursors have inflammatory biomarkers, possibly contributing to AD-related leaky gut although mechanisms of TMAO in $\mathrm{AD}$ remain unclear. The Western diet consists of low-fiber, high-fat, and high-protein foods, where it is common to eat fatty red meats and eggs that are rich in TMA and choline, thus increase TMAO production. This might partly explain higher AD cases in Western countries because Western diet is enriched with these ingredients. Additionally, TMAO-associated brain damage and cognitive deficits may be mediated by increased oxidative stress [17]. Because brain consumes a large amount of oxygen and the neurons have high metabolic rate, the neurons in the brain are at higher risk for oxidative stress in an average person. However, oxidative stress can be both cause and consequence of $\mathrm{AD}$ pathology. Inflammation instigates oxidative stress, which can lead to $A \beta$ and pTau accumulation; however, vice-versa is also possible in a destructive cycle. Additionally, oxidative stress can cause mitochon- 
drial dysfunction, and gut microbiota-derived metabolites influence mitochondrial functions. Oxidative stress can be modulated by diet, for example, high-fruit-andvegetable diets improve cognition, which is linked with decreased oxidative stress in elderly; whereas Western diet is not enriched with fruits and vegetables, which might also explain higher $\mathrm{AD}$ prevalence in Western countries.

\section{The Implication of Gut Microbiota in AD}

Gut microbiota is vital for our metabolic, immune, and brain health. Under healthy homeostatic conditions, the gut microbiota maintains a symbiotic relationship with the host and exerts important functions for host nutrition and metabolism, colonization resistance to pathogens, intestinal barrier integrity, and immune regulation. Gut microbiota has implications in brain diseases including depression, anxiety, hypertension, and Parkinson's disease. Recent studies also link gut microbiota with $\mathrm{AD}$; however, it should be noted that majority of these studies are cross-sectional investigations, and hence, more longitudinal intervention studies are needed to identify casual links between microbiota and $\mathrm{AD}$ pathology. We recently reported lower abundance of Bacteroidetes and higher abundance of Firmicutes and Proteobacteria (enterobacteriaceae) in MCI patients in addition to several differences at the genus level [7]. Other studies also reported lower diversity and lower Firmicutes-to-Bacteroidetes ratio in AD subjects [9], and lower Bacteroides in demented patients [18], while lower diversity, lower Firmicutes, and higher Proteobacteria were found in AD patients compared to MCI patients and normal subjects [19]. One study demonstrated involvement of butyrate-producing bacteria in cognitive function, where butyrate is classified as a post-biotic as it is the metabolic output (SCFA) of bacteria and has numerous benefits to the gut [20]. Interestingly, we also showed that a MD-ketogenic diet improved the $\mathrm{AD}$ biomarkers viz. amyloid and tau proteins in the cerebrospinal fluids of MCI patients, wherein these changes linked with increased gut butyrate [7].

Furthermore, an association of $A \beta$ accumulation with microbiota has been investigated. For instance, APP-PS1 mice, the most-widely used $\mathrm{AD}$ model, demonstrate $\mathrm{A} \beta$ accumulation in the brain in an age-dependent manner; however, its microbiota differs from wild-type mice. Further, mice transplanted with $\mathrm{AD}$ microbiota tend to have higher $A \beta$ accumulation. Further, $A \beta$ aggregation can be inhibited by microbiota-derived valerate and butyrate
[10]. Besides, bacterial endotoxins may also be involved in $\mathrm{AD}$-amyloidosis-related inflammation. For example, lipopolysaccharide (LPS), an outer cell wall component of Gram-negative bacteria that can be highly pro-inflammatory, can enhance $A \beta$ accumulation in brain and induce cognitive dysfunction [21]. AD patients show higher LPS levels in blood plasma [22], and neocortex and hippocampus [23]. LPS can also cause chronic neuroinflammation, nerve cell death in entorhinal cortex, and impairment of synaptic plasticity of neurons in hippocampus [24]. Specific bacteria, for example, Escherichia coli, Bacillus subtilis, Salmonella typhimurium, and Salmonella enterica can also produce amyloids [25]; however, these are not typical and consistent inhabitants of the human gut microbes. In addition, the relationship between $\mathrm{AD}$-associated amyloidosis versus bacterial amyloids remains unclear. Nevertheless, bacterial amyloid might activate specific signaling pathways involved in $\mathrm{AD}$ pathogenesis, hinting that the gut microbiota might exacerbate amyloidosis-associated inflammation. These studies link microbiota with the accumulation of amyloids via microbial extracellular components, pro-inflammatory factors, toxins, SCFAs, amyloid, and neurotransmitters; and underscore further investigation of links between gut microbiota and host neurodegenerative health [23]. Considering rapidly emerging studies, the future boom in $\mathrm{AD}$ prevention and cure research would foreseeably as well be directed toward microbiota research.

\section{Diet-Microbiota Interaction in the Amelioration of AD}

Given the emerging data on microbiota disparities between $\mathrm{AD}$ patients versus healthy subjects, researchers have started exploring ways to modulate the microbiome with the ambition to hopefully ameliorate AD pathology. Although gut microbiota can be manipulated through many methods including the use of probiotics, prebiotics, synbiotics, and antibiotics or change of diet, diet is the superlative modulator of gut microbiota. Dietary modulations focused on unsaturated fats, fruits and vegetables, and whole grains can confer benefits on AD-related cognitive health (Fig. 1). For instance, MD correlates with less brain atrophy in key-AD areas (indicating positive effect on $\mathrm{AD}$ pathology) [5], besides decreased inflammation, a major symptom of $\mathrm{AD}$, via increasing plasma carotenoids and decreasing C-reaction protein levels [4]. Higher consumption of fish products, which are rich in docosahexaenoic acid (a type of $n$ - 3 polyunsaturated fatty acid), has been linked with lower AD risks [3]. Further- 
more, fish, which is rich in vitamin D3, and dairy products, which are rich in vitamin $\mathrm{D}$, promote neural growth factor protein, which can protect against brain inflammation and aging [26]. Prompted by these ameliorative effects of MD against $\mathrm{AD}$, one human study examining the effects of MD-DASH Intervention for Neurodegenerative Delay (MIND) diet, which is a combination of MD with DASH diet (Dietary Approaches to Stop Hypertension), which is rich in fruits, vegetables, whole grains, low-fat dairy, and lean protein, demonstrated that MIND diet was more effective than MD or DASH alone, although all 3 diets demonstrated benefits at reducing $\mathrm{AD}$ pathology [6]. In addition to certain dietary lifestyles, individual foods can confer benefits against $\mathrm{AD}$ pathology. For instance, consumption of red wine has been shown to prevent the generation of $\mathrm{A} \beta$ peptides and reduce $\mathrm{AD}$ risk in mouse models [27]. Specifically, it has been reported that moderate alcohol consumption could decrease GABAergic sprouting of axon terminals that is potentially partially responsible for the neurodegeneration found in AD [28]. Flavonoids-rich foods, for example, black currants, grapes, citrus, and green tea, have been shown to inhibit $\mathrm{A} \beta$ deposition and prevent hyperphosphorylation of tau proteins, while improving other biomarkers of $\mathrm{AD}$ [29].

While the majority of studies examine the impact of healthy diet on the amelioration of AD pathology, several studies have explored the use of supplementation directly targeting the gut bacteria in $\mathrm{AD}$. A randomized double-blind controlled human trial looking at the effects of a 12-weeks consumption of a probiotic combination of Lactobacillus acidophilus, Lactobacillus casei, Bifidobacterium bifidum, and Lactobacillus fermentum, reported significant positive effects on cognitive functioning, although no effects on oxidative stress or inflammation was seen [30]. A mouse study examining the effects of the post-biotic sodium butyrate on $A \beta$ levels and behavioral symptoms in early AD mice found significant reduction in $A \beta$ levels and increase in behavioral responses after 12 -weeks of sodium butyrate supplementation [31]. Synbiotics, a combination of probiotics and prebiotics, also show benefits to $\mathrm{AD}$. One human study observed improvement in mental capabilities and reduced inflammation and oxidative stress in $\mathrm{AD}$ patients after consuming milk fermented with kefir grains (forming a symbiotic substance) for 90 days [32]. Taken together, the current literature on diet and supplementation influencing the gut microbiota and improve $\mathrm{AD}$ pathology is promising, but more studies are warranted. Additionally, research has targeted GABA by testing Tramiprosate, which is an analogue of GABA, and found that it showed promise in increasing the long-term potentiation that had been inhibited by $\mathrm{A} \beta$ in $\mathrm{AD}$ models as well as clinical trials [3335]. The drug trazodone, a serotonin agonist and reuptake inhibitor, has also indicated a correlation with delaying cognitive impairment in $\mathrm{AD}$ patients 2.6 times more than nontrazadone users [36].

\section{Conclusion}

$\mathrm{AD}$ is rapidly rising worldwide, but no cure is available hitherto, underscoring the need for effective strategies such as dietary factors to modulate $\mathrm{AD}$-related neuroinflammation and prevent or slow down AD progression. Current evidence shows that both pro-inflammatory and anti-inflammatory capacities of dietary components may play a role in $\mathrm{AD}$ management. Diets rich in simple sugars, saturated/trans fats, advanced glycation end-products, and processed meats may incite a pro-inflammatory influence on the brain of $\mathrm{AD}$ patients while potentially accelerating obesity, hypertension, dyslipidemia, atherosclerosis, and type-2 diabetes. Contrastingly, complex dietary patterns (e.g., MD, DASH, and MIND) rich in vegetables, fruits, salads, nuts, legumes, berries, polyunsaturated fatty acids, vitamins, flavonoids, polyphenols, probiotics/prebiotics, and whole grains may help in preventing or slowing down the cognitive decline and AD progression. Studies have suggested the involvement of gut microbiota in $\mathrm{AD}$ pathology via gut-brain axis. Diet strongly modulates the gut microbiota, which might be one of the mechanisms underlying the benefits of these dietary patterns in ameliorating $\mathrm{AD}$-related perturbations in gut-brain axis. Mechanistic understanding of interaction of lifestyle factors with $\mathrm{AD}$ may elucidate links between changing lifestyle patterns and increased $\mathrm{AD}$ prevalence while including factors such as microbiota that are crucial for this interaction between host lifestyle and health. Indeed, decoding the connections between diet, microbiota, lifestyle, and dementia would help revealing mechanisms underlying $\mathrm{AD}$ pathology while facilitating the discovery of novel strategies for prevention/treatment of $\mathrm{AD}$ and related dementia and cognitive decline.

\section{Conflict of Interest Statement}

Dr. Yadav is Chief Scientific Officer and Co-founder of the Post-biotics Inc.; however, he and other authors have no conflict of interest regarding this work and their duties. 


\section{Funding Sources}

The authors acknowledge the funding support from National Institutes of Health grant R01 AG018915 and the Pepper Older Americans for Independence Center (P30AG21332), and the Department of Defense funding W81XWH-18-PRARP-NIRA, and funds and services from the Center for Diabetes, Obesity, and Metabolism, Wake Forest Baptist Medical Center, and the National Center for Advancing Translational Sciences (NCATS), the National Institutes of Health-funded Wake Forest Clinical and Translational Science Institute (WF CTSI) through Grant Award Number UL1TR001420.

\section{Author Contributions}

H.K. and R.N.: collected literature, wrote and revised the manuscript; H.Y.: supervised, reviewed, and revised the manuscript; H.K., R.N., and H.Y.: approved the final version of the manuscript.

\section{References}

1 Prince M, Bryce R, Albanese E, Wimo A, Ribeiro W, Ferri CP. The global prevalence of dementia: a systematic review and metaanalysis. Alzheimers Dement. 2013 Jan;9(1):63-e2.

2 Alonso R, Pisa D, Fernández-Fernández AM, Carrasco L. Infection of fungi and bacteria in brain tissue from elderly persons and patients with Alzheimer's disease. Front Aging Neurosci. 2018;10:159.

3 Szczechowiak K, Diniz BS, Leszek J. Diet and Alzheimer's dementia: nutritional approach to modulate inflammation. Pharmacol Biochem Behav. 2019 Sep;184:172743.

4 Blum S, Aviram M, Ben-Amotz A, Levy Y. Effect of a mediterranean meal on postprandial carotenoids, paraoxonase activity and C-reactive protein levels. Ann Nutr Metab. 2006; 50(1):20-4.

5 Mosconi L, Murray J, Tsui WH, Li Y, Davies $\mathrm{M}$, Williams S, et al. Mediterranean diet and magnetic resonance imaging-assessed brain atrophy in cognitively normal individuals at risk for Alzheimer's disease. J Prev Alzheimers Dis. 2014 Jun;1(1):23-32.

6 Morris MC, Tangney CC, Wang Y, Sacks FM, Bennett DA, Aggarwal NT. MIND diet associated with reduced incidence of Alzheimer's disease. Alzheimers Dement. 2015 Sep;11(9): 1007-14.

7 Nagpal R, Neth BJ, Wang S, Craft S, Yadav H. Modified mediterranean-ketogenic diet modulates gut microbiome and short-chain fatty acids in association with Alzheimer's disease markers in subjects with mild cognitive impairment. EBioMedicine. 2019 Sep;47:52942.

8 Bhattacharjee S, Lukiw WJ. Alzheimer's disease and the microbiome. Front Cell Neurosci. 2013;7:153.

9 Vogt NM, Kerby RL, Dill-McFarland KA, Harding SJ, Merluzzi AP, Johnson SC, et al. Gut microbiome alterations in Alzheimer's disease. Sci Rep. 2017 Oct 19;7(1):13537.

10 Ho L, Ono K, Tsuji M, Mazzola P, Singh R, Pasinetti GM. Protective roles of intestinal microbiota derived short chain fatty acids in Alzheimer's disease-type beta-amyloid neuropathological mechanisms. Expert Rev Neurother. 2018 Jan;18(1):83-90.
11 Potter H, Granic A, Caneus J. Role of trisomy 21 mosaicism in sporadic and familial Alzheimer's disease. Curr Alzheimer Res. 2016; 13(1):7-17.

12 Fuster-Matanzo A, Llorens-Martín M, Hernández F, Avila J. Role of neuroinflammation in adult neurogenesis and Alzheimer disease: therapeutic approaches. Mediators Inflamm. 2013;2013:260925.

13 Manoharan S, Guillemin GJ, Abiramasundari RS, Essa MM, Akbar M, Akbar MD. The role of reactive oxygen species in the pathogenesis of Alzheimer's disease, Parkinson's disease, and Huntington's disease: a mini review. Oxid Med Cell Longev. 2016;2016:8590578.

14 Atri A. The Alzheimer's disease clinical spectrum: diagnosis and management. Med Clin North Am. 2019 Mar;103(2):263-93.

15 Thevaranjan N, Puchta A, Schulz C, Naidoo A, Szamosi JC, Verschoor CP, et al. Age-associated microbial dysbiosis promotes intestinal permeability, systemic inflammation, and macrophage dysfunction. Cell Host Microbe. 2017 Apr 12;21(4):455-e4.

16 Vogt NM, Romano KA, Darst BF, Engelman CD, Johnson SC, Carlsson CM, et al. The gut microbiota-derived metabolite trimethylamine $\mathrm{N}$-oxide is elevated in Alzheimer's disease. Alzheimers Res Ther. 2018 Dec 22;10(1): 124.

17 Li D, Ke Y, Zhan R, Liu C, Zhao M, Zeng A, et al. Trimethylamine- $\mathrm{N}$-oxide promotes brain aging and cognitive impairment in mice. Aging Cell. 2018 Aug;17(4):e12768.

18 Saji N, Niida S, Murotani K, Hisada T, Tsuduki T, Sugimoto T, et al. Analysis of the relationship between the gut microbiome and dementia: a cross-sectional study conducted in Japan. Sci Rep. 2019 Jan 30;9(1):1008.

19 Liu P, Wu L, Peng G, Han Y, Tang R, Ge J, et al. Altered microbiomes distinguish Alzheimer's disease from amnestic mild cognitive impairment and health in a Chinese cohort. Brain Behav Immun. 2019 Aug;80:633-43.

20 Nguyen TTT, Fujimura Y, Mimura I, Fujii Y, Nguyen NL, Arakawa K, et al. Cultivable butyrate-producing bacteria of elderly Japanese diagnosed with Alzheimer's disease. J Microbiol. 2018 Oct;56(10):760-71.
21 Zhan X, Stamova B, Jin LW, DeCarli C, Phinney B, Sharp FR. Gram-negative bacterial molecules associate with Alzheimer disease pathology. Neurology. 2016 Nov 29;87(22): 2324-32.

22 Zhang R, Miller RG, Gascon R, Champion S, Katz J, Lancero M, et al. Circulating endotoxin and systemic immune activation in sporadic amyotrophic lateral sclerosis (sALS). J Neuroimmunol. 2009 Jan 3;206(1-2):121-4.

23 Zhao Y, Jaber V, Lukiw WJ. Secretory products of the human GI tract microbiome and their potential impact on Alzheimer's disease (AD): detection of lipopolysaccharide (LPS) in AD hippocampus. Front Cell Infect Microbiol. 2017;7:318.

24 Hauss-Wegrzyniak B, Wenk GL. Beta-amyloid deposition in the brains of rats chronically infused with thiorphan or lipopolysaccharide: the role of ascorbic acid in the vehicle. Neurosci Lett. 2002 Apr 5;322(2): 75-8.

25 Schwartz K, Boles BR. Microbial amyloids: functions and interactions within the host. Curr Opin Microbiol. 2013 Feb;16(1):93-9.

26 Brown J, Bianco JI, McGrath JJ, Eyles DW. 1,25-dihydroxyvitamin D3 induces nerve growth factor, promotes neurite outgrowth and inhibits mitosis in embryonic rat hippocampal neurons. Neurosci Lett. 2003 Jun 5; 343(2):139-43.

27 Wang J, Ho L, Zhao Z, Seror I, Humala N, Dickstein DL, et al. Moderate consumption of cabernet sauvignon attenuates abeta neuropathology in a mouse model of Alzheimer's disease. Faseb j. 2006 Nov;20(13):231320.

28 Frisardi V, Panza F, Seripa D, Imbimbo BP, Vendemiale G, Pilotto A, et al. Nutraceutical properties of mediterranean diet and cognitive decline: possible underlying mechanisms. J Alzheimers Dis. 2010;22(3):715-40.

29 Ayaz M, Sadiq A, Junaid M, Ullah F, Ovais M, Ullah I, et al. Flavonoids as prospective neuroprotectants and their therapeutic propensity in aging associated neurological disorders. Front Aging Neurosci. 2019;11:155. 
30 Akbari E, Asemi Z, Daneshvar Kakhaki R, Bahmani F, Kouchaki E, Tamtaji OR, et al. Effect of probiotic supplementation on cognitive function and metabolic status in $\mathrm{Al}$ zheimer's disease: a randomized, doubleblind and controlled trial. Front Aging Neurosci. 2016;8:256.

31 Fernando WMADB, Martins IJ, Morici M, Bharadwaj P, Rainey-Smith SR, Lim WLF, et al. Sodium butyrate reduces brain amyloid- $\beta$ levels and improves cognitive memory performance in an Alzheimer's disease transgenic mouse model at an early disease stage. J Alzheimers Dis. 2020;74(1):91-9.
32 Ton AMM, Campagnaro BP, Alves GA, Aires $\mathrm{R}$, Côco LZ, Arpini CM, et al. Oxidative stress and dementia in Alzheimer's patients: effects of synbiotic supplementation. Oxid Med Cell Longev. 2020;2020:2638703.

33 Aisen PS, Saumier D, Briand R, Laurin J, Gervais $\mathrm{F}$, Tremblay $\mathrm{P}$, et al. A phase II study targeting amyloid-beta with 3APS in mild-tomoderate Alzheimer disease. Neurology. 2006 Nov 28;67(10):1757-63.

34 Sabbagh MN. Drug development for Alzheimer's disease: where are we now and where are we headed? Am J Geriatr Pharmacother. 2009 Jun;7(3):167-85.
35 Caltagirone C, Ferrannini L, Marchionni N, Nappi G, Scapagnini G, Trabucchi M. The potential protective effect of tramiprosate (homotaurine) against Alzheimer's disease: a review. Aging Clin Exp Res. 2012 Dec;24(6): 580-7.

36 La AL, Walsh CM, Neylan TC, Vossel KA, Yaffe K, Krystal AD, et al. Long-term trazodone use and cognition: a potential therapeutic role for slow-wave sleep enhancers. J Alzheimers Dis. 2019;67(3):911-21. 